\title{
Modeling granular material blending in a Tote blender using a finite element method and advection-diffusion equation multi-scale model
}

\author{
Yu Liu ${ }^{\mathrm{a}}$, Andrew Thomas Cameron ${ }^{\mathrm{a}}$, Marcial Gonzalez ${ }^{\mathrm{a}}$ and Carl Wassgren, ${ }^{\mathrm{a}, \mathrm{b}}{ }^{*}$ \\ ${ }^{a}$ School of Mechanical Engineering, 585 Purdue Mall, Purdue University, West Lafayette, IN \\ 47907-2088, U.S.A. \\ ${ }^{\mathrm{b}}$ Department of Industrial and Physical Pharmacy (by courtesy), 575 Stadium Mall Drive, \\ Purdue University, West Lafayette, IN 47907-2091, U.S.A. \\ * Corresponding author at: School of Mechanical Engineering, Purdue University, West \\ Lafayette, IN 47907-2088, U.S.A., Tel.: +1 765494 5656, E-mail address: \\ wassgren@purdue.edu (C. Wassgren).
}

\begin{abstract}
A multi-scale model is presented for predicting the magnitude and rate of powder mixing in a Tote blender. The model combines particle diffusion correlations calibrated from experiments with transient advective flow field information from finite element method simulations. Predictions of the mixing rate from the multi-scale model compare well quantitatively to published experimental data. The multi-scale model, since it does not directly model individual particles, is expected to scale well to systems of industrial interest.
\end{abstract}

Keywords: Blending; Granular material; Finite element method; Multi-scale model

\section{$1 \quad$ Introduction}

Granular material blending plays an important role in many industries ranging from those that manufacture pharmaceuticals to those producing agrochemicals. The ability to create homogeneous powder blends can be critical to the final product quality. For example, insufficient blending of a pharmaceutical formulation may have serious consequences on product efficacy and safety.

A wide variety of blenders are available in the marketplace [1,2]. However, predictive engineering design of industrial powder blenders remains underdeveloped due to the lack of quantitative modeling tools. As a result, design and scale-up of blending equipment often relies on empirical studies. Discrete element method (DEM) computational models are considered state-of-the-art for predicting blending of particulate materials; however, this modeling approach is not well-suited for modeling industrial-scale blenders due to computational limitations [3]. Moreover, determining particle-level input parameters to use in such models is challenging and not widely agreed upon [4-6].

In recent work by Liu et al. [7], a multi-scale model was developed by combining particle diffusion coefficient correlations with advective flow field information from finite element method (FEM) simulations. The model was able to quantitatively predict the magnitude and rate of powder blending in a two-dimensional rotating drum blender and was computationally faster than DEM simulations. The current work extends this multi-scale modeling approach to a more complex, three-dimensional blender geometry with transient powder dynamics. 


\section{$2 \quad$ Background}

Most models that track the spatial and temporal evolution of particle blending can be categorized as being either of the discrete or continuum type. Discrete algorithms model the dynamics of each particle individually while continuum algorithms ignore individual particles and instead assume that the particle assembly is a continuum.

The computational discrete element method (DEM) has been used extensively for predicting particle blending dynamics and is considered state-of-the-art [8-11]. Although these studies have provided valuable qualitative insight, they are limited in their quantitative predictions by computational requirements. Even a small, lab-scale blender containing $100 \mu \mathrm{m}$ particles contains more than $10^{9}$ particles, far exceeding what is possible to model with standard computational tools. Typically, modelers will increase the particle size or decrease the workspace size in order to reduce the number of particles that must be modeled; however, previous work $[8,12]$ has shown that particle size can have an influence on the rate and extent of blending. Hence, DEM may not produce quantitatively accurate results for systems of industrial interest.

Analytical continuum models have also been proposed for modeling mixing and segregation [13-15]. To be tractable analytically, these early models oversimplified the effects of key physical properties and the predictions from these purely theoretical studies were not very accurate. To improve the accuracy of these models, recent works $[16,17]$ combined DEMcalculated correlations for particle diffusion and segregation at a local scale with analyticallyderived flow fields. These models have been shown to provide quantitatively accurate predictions; however, since they depend on analytical solutions for the macroscopic flow field, their use is restricted to simple two-dimensional geometries, such as a simple heap flow.

Recently, researchers have utilized multi-scale modeling approaches to make quantitative blending predictions. Bertuola et al. [18] combined velocity field data predicted from finite element method (FEM) simulations, which treat the granular material as a generalized Newtonian fluid, with local-scale mixing and segregation relations derived from previous works $[16,19]$. Key model parameters needed to be fit to the experimental data for quantitative agreement. Bai et al. [20] used an FEM model assuming Mohr-Coulomb constitutive behavior to predict the velocity field in a cylindrical, bladed mixer, which was shown to be qualitatively accurate compared to DEM simulations. They also predicted the degree of blending by assuming convective mixing only (neglecting diffusive mixing), but observed a dependence of the mixing rate on FEM mesh size. Liu et al. [7] also investigated particle blending using a multi-scale modeling approach. An FEM model, which assumed Mohr-Coulomb material behavior, was used to predict the velocity field in a two-dimensional rotating drum. This information was then combined with particle diffusion correlations. The model was shown to quantitatively predict the magnitude and rate of blending when compared to DEM simulations without the need for backfitted parameters.

The current work extends the work by Liu et al. [7] to investigate blending in a more industrially-relevant Tote blender. Several key implementation details are also different. First, the model utilizes transient velocity field information from the FEM simulations instead of a 
steady velocity field. Second, the governing equations are extended to three dimensions instead of two dimensions. Together, these two modifications greatly increase the flexibility of the model. In addition, rotating drum experiments are used to calibrate the diffusion coefficient used in the multi-scale model and the model predictions are compared against published experimental results. Section 3 of this paper introduces the FEM modeling approach and implementation for the current work. Section 4 describes the advection-diffusion equation used in the multi-scale model and the numerical method used to solve it. Section 5 describes the material calibration methods and experiments. And in Section 6, comparisons are made between published experimental results and the multi-scale model predictions.

\section{$3 \quad$ Finite element method model}

A three-dimensional, coupled Eulerian-Lagrangian, FEM model is used in the present work to provide predictions of the advective flow field in a Tote blender. Previous works [21-25] have shown that FEM models can accurately simulate granular material behavior well, including advective flow fields [21,22]. Details of the model implementation can be found in previous work [7]. The following sub-sections describe the model geometry, boundary conditions, and initial conditions.

\subsection{Model geometry and boundary conditions}

The commercial FEM package Abaqus/Explicit v6.14 is used to perform the bulk flow simulations. The geometry of the simulated Tote blender is based on the experiments by Sudah et al. [26]. Those experiments were carried out in a 14-L GEI Gallay Tote blender. Details of the geometry and dimensions of the blender are presented in their work [26]. For convenience, the dimensions are also shown in Figure 1.

a)

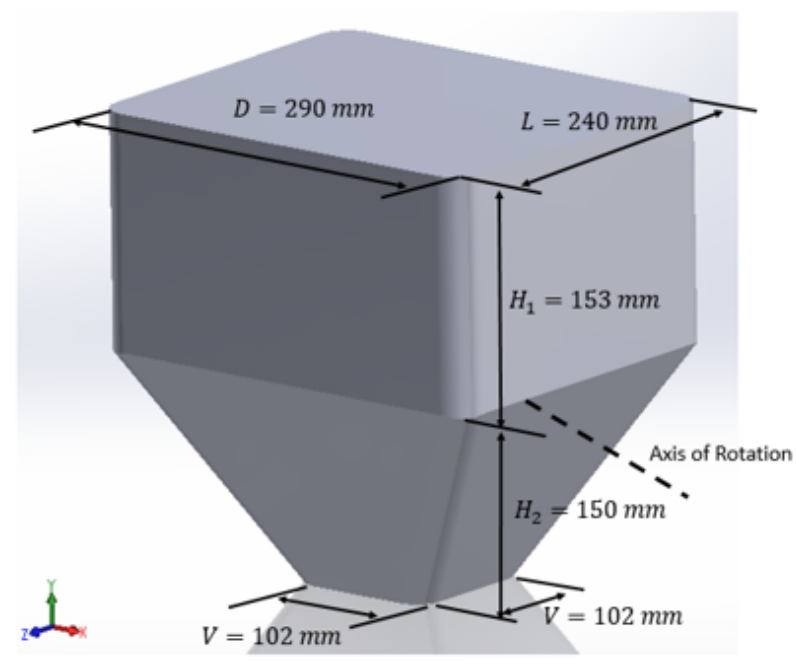

b)

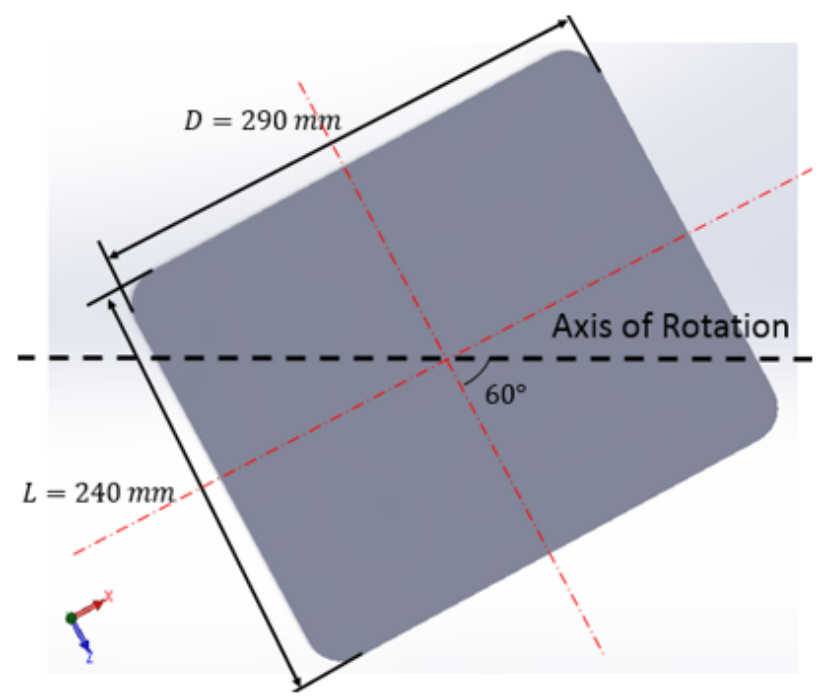

Figure 1. Dimensions of the GEA Gallay Tote blender used in the FEM simulation.

A Mohr-Coulomb elastoplastic model is used in the current work to describe the stress-strain behavior of the particulate material. This model has been shown previously to accurately describe granular flow fields [21,22]. The material properties needed in the model are bulk 
density, Young's modulus, Poisson's ratio, and material internal friction angle. The methodology for obtaining those material properties was described in detail in previous work [7].

Boundary conditions applied in the model include the material-wall friction angle, the rotational speed along the axis of rotation, and the gravitational acceleration $\left(g=9.8 \mathrm{~m} / \mathrm{s}^{2}\right.$ directed in the negative $y$ direction in Figure 1).

\subsection{Initial conditions}

The coupled Eulerian-Lagrangian approach implemented in Abaqus is adopted in the current model. Details of this approach were described in previous work [7]. As shown in Figure 2, the Eulerian mesh covers the entire material domain and ensures that no material leaks outside of the mesh. The Eulerian Volume Fraction (EVF) value is used to determine the volume of material within each element. A value of $\mathrm{EVF}=0$ indicates that no material is present in the element while $\mathrm{EVF}=1$ indicates that the element is completely filled with material.

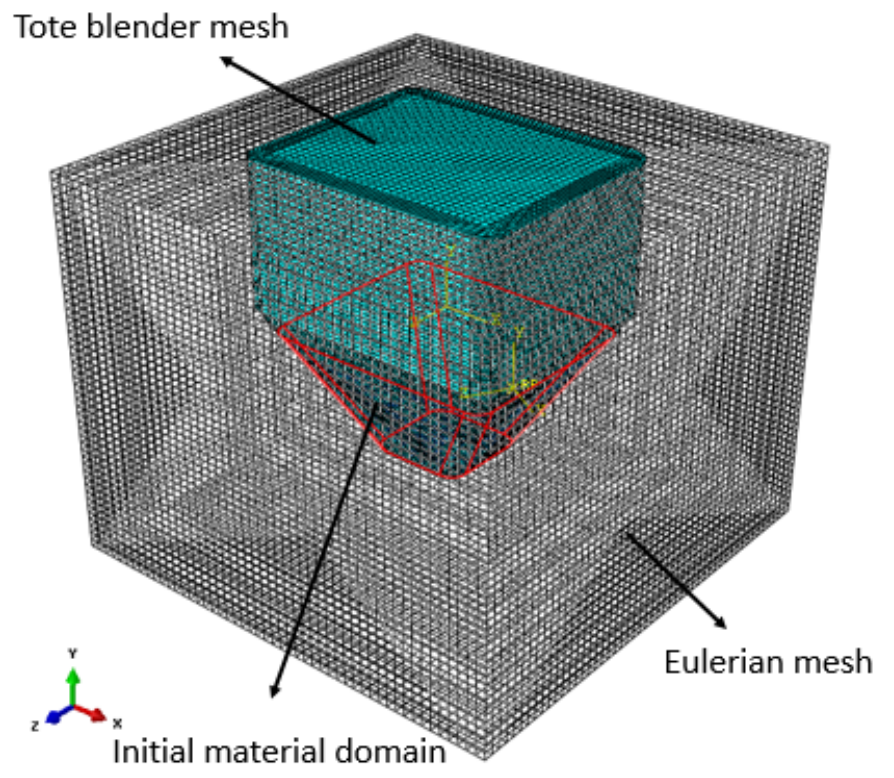

Figure 2. A schematic of the FEM model domain. The Eulerian mesh is shown in grey, the Tote blender mesh is shown in blue, and the outlines of the initial material domain are shown in red.

The initial bed state is generated by filling a fraction of the elements with material. Details of the process can be found in previous work [7]. For monodisperse mixing, material loadings were achieved by assigning user-defined field variables at each material point to represent the initial material concentration. Details of this field variable approach can be found in the Abaqus documentation [27]. An example of left-right initial loading is shown in Figure 3. The color represents a field variable value from 0 (blue) to 1 (red). For monodisperse mixing with materials $\mathrm{A}$ and $\mathrm{B}$, the field variable represents the material concentration of $\mathrm{A}$. A field variable value of one indicates that the element is completely filled with material $\mathrm{A}$, while a value of zero indicates that the element is completely filled with material $\mathrm{B}$. 
a)

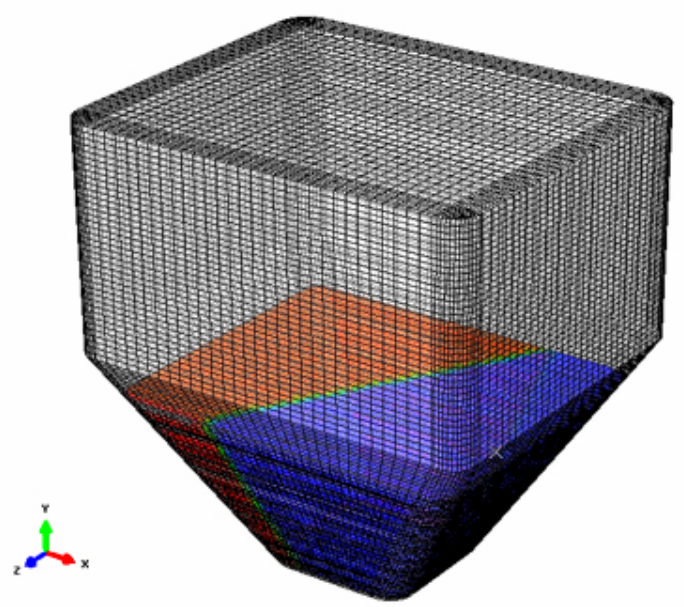

b)

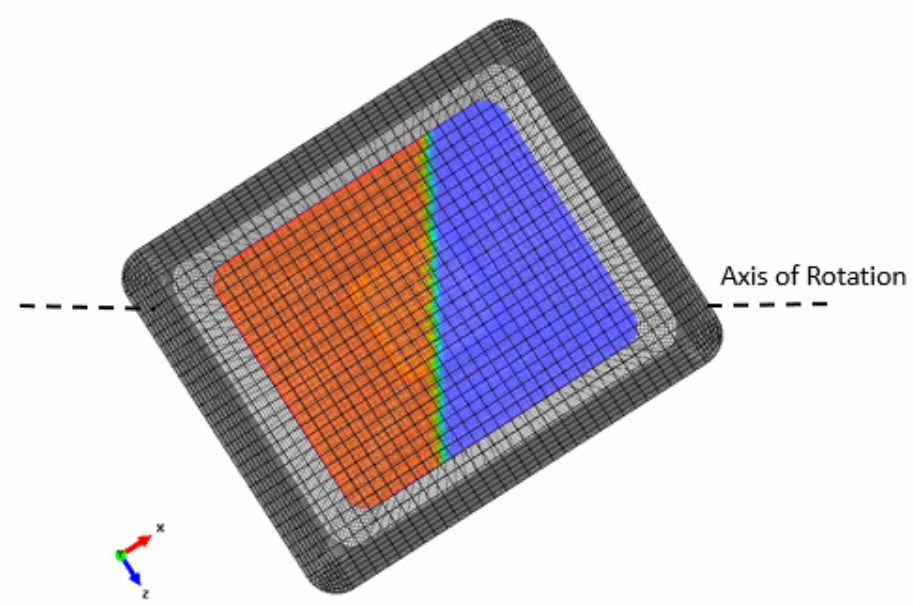

Figure 3. Initial material concentration for a simulation with left-right loading. The color represents field variable value from zero (blue) to one (red). The materials in this simulation have homogeneous properties, except for color.

The simulation process consists of two steps. First, the material is allowed to settle as the gravitational acceleration is slowly increased from zero to its final value. The blender remains stationary during this step. This procedure is used to eliminate the transient oscillations of material as it settles and results in a stable bed compressed under its own weight. Next, the blender is allowed to rotate immediately at the rotation speed and the simulation is considered started.

\section{$4 \quad$ The multi-scale blending model}

The current multi-scale model is extended from a previously published two-dimensional model [7]. Details of the model description and development can be found in this previous work. The following sub-sections summarize the main aspects of the model and highlight its extension to transient, three-dimensional problems.

\subsection{Advection-diffusion equation}

The advection-diffusion equation is used in the current work to model the spatiotemporal evolution of the concentration of a particular material species, $c$. The governing equation is derived as,

$$
\frac{\partial c}{\partial t}=\nabla \cdot(\boldsymbol{D} \nabla c)-\nabla \cdot(\boldsymbol{v} c)
$$

where $c$ is the local concentration of a particular species of material, $\boldsymbol{D}$ is the diffusion coefficient tensor for that species, and $v$ is the local advective velocity vector. Using the local mass conservation equation and assuming an incompressible material, i.e.,

$\nabla \cdot v=0$

the governing equation can be written in index notation form as,

$\frac{\partial c}{\partial t}=\frac{\partial}{\partial x_{i}}\left(D_{i j} \frac{\partial c}{\partial x_{j}}\right)-v_{i} \frac{\partial c}{\partial x_{i}}$. 
The self-diffusion coefficient $\boldsymbol{D}$ is a tensor quantity with components $D_{i j}$, and with off-diagonal components $D_{i j}(i \neq j)$ an order of magnitude smaller than the diagonal components $D_{i j}(i=j)$ [7]. By neglecting off-diagonal components of the self-diffusion coefficient tensor, the index notation form of Eq. (4.3) in three-dimensional form is

$$
\frac{\partial c}{\partial t}=\left(\frac{\partial D_{x x}}{\partial x}-v_{x}\right) \frac{\partial c}{\partial x}+D_{x x} \frac{\partial^{2} c}{\partial x^{2}}+\left(\frac{\partial D_{y y}}{\partial y}-v_{y}\right) \frac{\partial c}{\partial y}+D_{y y} \frac{\partial^{2} c}{\partial y^{2}}+\left(\frac{\partial D_{z z}}{\partial z}-v_{z}\right) \frac{\partial c}{\partial z}+D_{z z} \frac{\partial^{2} c}{\partial z^{2}} .
$$

The self-diffusion coefficient $\boldsymbol{D}$ is also proportional to the local shear rate $\dot{\gamma}$ and the local mean particle diameter $\bar{d}$ [7]. The particle diffusivity is approximately 1.9 times larger along the mean flow direction than it is in the perpendicular direction, according to work by Utter et al. [28]. Thus, the shear rate-dependent diffusion coefficient $\boldsymbol{D}$ can be written as,

$$
\begin{aligned}
& D_{x x}=k_{1} \dot{\gamma}_{x} \bar{d}^{2}+k_{2}\left(\dot{\gamma}_{y}+\dot{\gamma}_{z}\right) \bar{d}^{2} \\
& D_{\mathrm{yy}}=k_{1} \dot{\gamma}_{y} \bar{d}^{2}+k_{2}\left(\dot{\gamma}_{x}+\dot{\gamma}_{z}\right) \bar{d}^{2}, \\
& D_{z z}=k_{1} \dot{\gamma}_{z} \bar{d}^{2}+k_{2}\left(\dot{\gamma}_{x}+\dot{\gamma}_{y}\right) \bar{d}^{2}
\end{aligned}
$$

where $\dot{\gamma}_{x}=\left(\left|\partial v_{y} / \partial x\right|+\left|\partial v_{z} / \partial x\right|\right), \dot{\gamma}_{y}=\left(\left|\partial v_{x} / \partial y\right|+\left|\partial v_{z} / \partial y\right|\right)$, and $\dot{\gamma}_{z}=\left(\left|\partial v_{x} / \partial z\right|+\left|\partial v_{y} / \partial z\right|\right)$. The constant $k_{1}$ can be found from experiments or small-scale DEM simulations, with $k_{2}=1.9 k_{1}$.

\subsection{Numerical method}

A finite difference method using a central explicit scheme is used to solve Eq. (4.4) due to its simplicity and computational efficiency [7]. Other numerical methods are also available to solve the system of partial differential equations, such as the finite element method, finite volume method, domain decomposition method, and the matrix mapping method [16,17].

A second-order Tylor Lax-Wendroff scheme is used to generate the finite difference expression of the governing equation [7],

$$
\begin{aligned}
& c_{i j k}^{n+1}=c_{i j k}^{n}-\left[v_{x} \Delta_{x 0} c_{i j k}^{n}-\left(\frac{1}{2} v_{x}^{2}+\mu_{x}\right) \delta_{x}^{2} c_{i j k}^{n}\right]-\left[v_{y} \Delta_{y 0} c_{i j k}^{n}-\left(\frac{1}{2} v_{y}^{2}+\mu_{y}\right) \delta_{y}^{2} c_{i j k}^{n}\right] \\
& -\left[v_{z} \Delta_{z 0} c_{i j k}^{n}-\left(\frac{1}{2} v_{z}^{2}+\mu_{z}\right) \delta_{z}^{2} c_{i j k}^{n}\right]+v_{x} v_{y} \Delta_{x 0} \Delta_{y 0} c_{i j k}^{n}+v_{y} v_{z} \Delta_{y 0} \Delta_{z 0} c_{i j k}^{n}+v_{z} v_{x} \Delta_{z 0} \Delta_{x 0} c_{i j k}^{n}
\end{aligned},
$$

where,

$$
\begin{aligned}
& v_{x}=\frac{\left[v_{x}+\Delta_{x 0}\left(D_{x x}\right)_{i j k}\right] \Delta t}{\Delta x}, \\
& v_{y}=\frac{\left[v_{y}+\Delta_{y 0}\left(D_{y y}\right)_{i j k}\right] \Delta t}{\Delta y}, \\
& v_{z}=\frac{\left[v_{z}+\Delta_{z 0}\left(D_{z z}\right)_{i j k}\right] \Delta t}{\Delta z},
\end{aligned}
$$




$$
\begin{aligned}
& \mu_{x}=\left(D_{x x}\right)_{i j k} \frac{\Delta t}{\Delta x^{2}}, \\
& \mu_{y}=\left(D_{y y}\right)_{i j k} \frac{\Delta t}{\Delta y^{2}}, \\
& \mu_{z}=\left(D_{z z}\right)_{i j k} \frac{\Delta t}{\Delta z^{2}}, \\
& \Delta_{x 0} c_{i j k}^{n}=\frac{c_{i, j+1, k}^{n}-c_{i, j-1, k}^{n}}{2}, \\
& \Delta_{y 0} c_{i j k}^{n}=\frac{c_{i+1, j, k}^{n}-c_{i-1, j, k}^{n}}{2}, \\
& \Delta_{z 0} c_{i j k}^{n}=\frac{c_{i, j, k+1}^{n}-c_{i, j, k-1}^{n}}{2}, \\
& \delta_{x}^{2} c_{i j k}^{n}=c_{i, j+1, k}^{n}-2 c_{i j k}^{n}+c_{i, j-1, k}^{n}, \\
& \delta_{y}^{2} c_{i j k}^{n}=c_{i+1, j, k}^{n}-2 c_{i j k}^{n}+c_{i-1, j, k}^{n}, \\
& \delta_{z}^{2} c_{i j k}^{n}=c_{i, j, k+1}^{n}-2 c_{i j k}^{n}+c_{i, j, k-1}^{n} \text {, } \\
& \Delta_{x 0} \Delta_{y 0} c_{i j k}^{n}=\frac{\left(c_{i+1, j+1, k}^{n}-c_{i-1, j+1, k}^{n}-c_{i+1, j-1, k}^{n}+c_{i-1, j-1, k}^{n}\right)}{4}, \\
& \Delta_{y 0} \Delta_{z 0} c_{i j k}^{n}=\frac{\left(c_{i+1, j, k+1}^{n}-c_{i-1, j, k+1}^{n}-c_{i+1, j, k-1}^{n}+c_{i-1, j, k-1}^{n}\right)}{4}, \\
& \Delta_{z 0} \Delta_{x 0} c_{i j k}^{n}=\frac{\left(c_{i, j+1, k+1}^{n}-c_{i, j-1, k+1}^{n}-c_{i, j+1, k-1}^{n}+c_{i, j-1, k-1}^{n}\right)}{4}, \\
& \Delta_{x 0}\left(D_{x x}\right)_{i j k}=\frac{\left(D_{x x}\right)_{i, j+1, k}-\left(D_{x x}\right)_{i, j-1, k}}{2}, \\
& \Delta_{y 0}\left(D_{y y}\right)_{i j k}=\frac{\left(D_{y y}\right)_{i+1, j, k}-\left(D_{y y}\right)_{i-1, j, k}}{2} \text {, and } \\
& \Delta_{z 0}\left(D_{z z}\right)_{i j k}=\frac{\left(D_{z z}\right)_{i, j, k+1}-\left(D_{z z}\right)_{i, j, k-1}}{2} .
\end{aligned}
$$

This finite difference formula is illustrated using the computational molecule shown in Figure 4. Details of the Taylor series expansion and stability of the numerical computations can be found in the previous work [7]. 


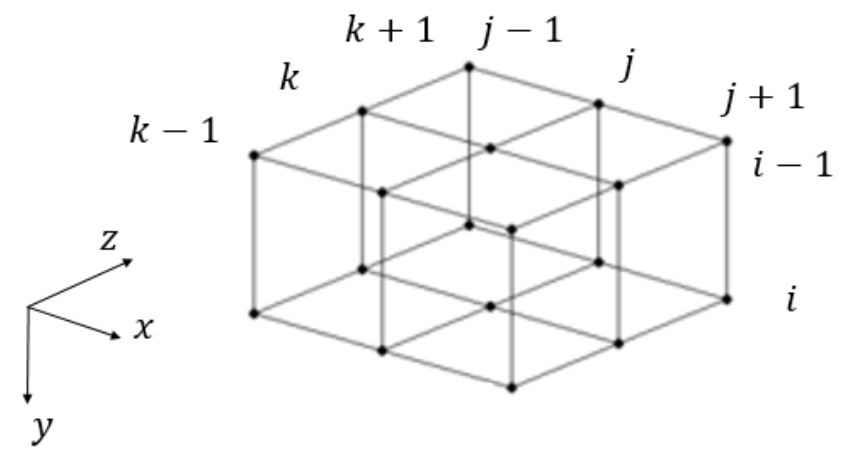

Figure 4. Computational molecule for the second-order, three-dimensional Taylor Lax-Wendroff scheme.

It should be emphasized that transient velocity fields are included in the solution to the advection-diffusion equation, Eq. (4.4), by extending the work by Liu et al. [7]. Therefore, a C++ post-processing script was developed to process the FEM output files (.obd files [27]) and handle the large amount of data from these simulations.

As mentioned in Section 3, the initial particle concentrations used in the model are determined by the loading conditions, and the user-defined field variable used in the FEM simulation is set accordingly, as in Figure 3. Since transient velocity fields are used in the current model, the boundaries of the material domain change throughout the entire computation. These boundaries are computed within the FEM simulation from the EVF values and, similarly, are determined by the advection-diffusion solver, after reading EVF values from the FEM output file at each time step. Specifically, the advection-diffusion boundary conditions at the free surface are enforced by setting the material concentration of the boundary node equal to the value of the node that is one grid point inward in the direction normal to the surface. This approach is similar to the polynomial fitting approach often used in computational fluid dynamics (CFD) [29].

Next, a MATLAB program is used to iterate the finite difference form of the advection-diffusion equation given in Eq. (4.6). After generating initial particle concentrations using the extracted field variable values, the material concentration evolution is iterated using the transient velocity field obtained from the FEM simulation for each time step. A threshold is set to ensure the material concentration value remains between zero and one, and a small time step is carefully chosen to ensure the stability of the explicit scheme. Details of the iteration algorithm were presented in the work by Liu et al. [7].

To achieve a converged result, the number of elements used in the advection-diffusion MATLAB program should be much larger than the number of elements in the FEM simulation. Hence, a linear interpolation algorithm is introduced in the MATLAB program to generate enough elements and ensure convergence. The MATLAB numerical algorithms are parallelized to divide the computational domain onto different cores. By using the full processing power of an eight-core desktop, the iteration runs approximately 5-8 times faster than on a single core. 


\section{$5 \quad$ Calibration of material properties}

\subsection{Material properties for the FEM simulation}

The published experimental work for monodisperse particle mixing by Sudah et al. [26] was used to validate the multi-scale model predictions. Those experiments utilized a 14-L GEI Gallay Tote blender containing 12-mm diameter glass beads of two different colors.

The material density $\rho$, elastic modulus $E$, and Poisson's ratio $v$ are known to have little influence on the material flow behavior [7]. Moreover, the mixing process was shown to be unaffected by the wall friction angle $\phi_{w}$ within a rotating blender since the free surface angle remains constant. Hence, the material density, elastic modulus, Poisson's ratio, and wall friction angle used in the current FEM simulation are all based on values from previous work for hard spheres [7]. In addition, the Mohr-Coulomb dilation angle of the material was set to $0.1^{\circ}$, since the dilation of cohesionless granular materials is usually small.

The internal friction angle has been shown to influence the mixing rate [7] and, hence, must be calibrated. Previous work has shown that the internal friction angle is not sensitive to particle size or consolidation stress for the same material [30-32]. Hence, 1-mm diameter glass beads, as opposed to the $12 \mathrm{~mm}$ beads used in the Sudah et al. experiments [26], were used in the current work to calibrate the internal friction angle. A Schulze Ring Shear Tester (Model RST-XS) was used to make these measurements, obtaining values similar to those reported in the literature [33,34]. A summary of all of the material parameters used in the FEM simulation is given in Table 1. It is worth noting that these parameters are found from independent, standard material tests, rather than being back-fit to match experimental data with the multi-scale blending simulation results.

Table 1. Parameters used in the FEM simulation.

\begin{tabular}{ll}
\hline Parameter & Value \\
\hline Material density $\left(\mathrm{kg} / \mathrm{m}^{3}\right)$ & 1500 \\
Young's modulus (MPa) & 3.65 \\
Poisson's ratio (-) & 0.065 \\
Internal friction angle (degree) & 27.1 \\
Cohesion (Pa) & 87 \\
Dilation angle (degree) & 0.1 \\
Wall friction coefficient (-) & 0.324 \\
\hline
\end{tabular}

\subsection{Experimental calibration of the diffusion constant}

One significant parameter needed in the multi-scale model is the spanwise diffusion constant $k_{1}$. To calibrate $k_{1}$ for glass beads, a lab-scale rotating drum experiment was performed. A photograph of the experiment setup is shown in Figure 5. An acrylic circular drum of diameter $D$ $=150 \mathrm{~mm}$ and width $W=50 \mathrm{~mm}$ was used to contain the material, and two shafts were used to stabilize and rotate the drum. The driving torque was provided by a gear motor that rotated one of the shafts. Rubber bands covered the shafts to prevent slipping. Values for the experiment parameters are listed in Table 2 . 


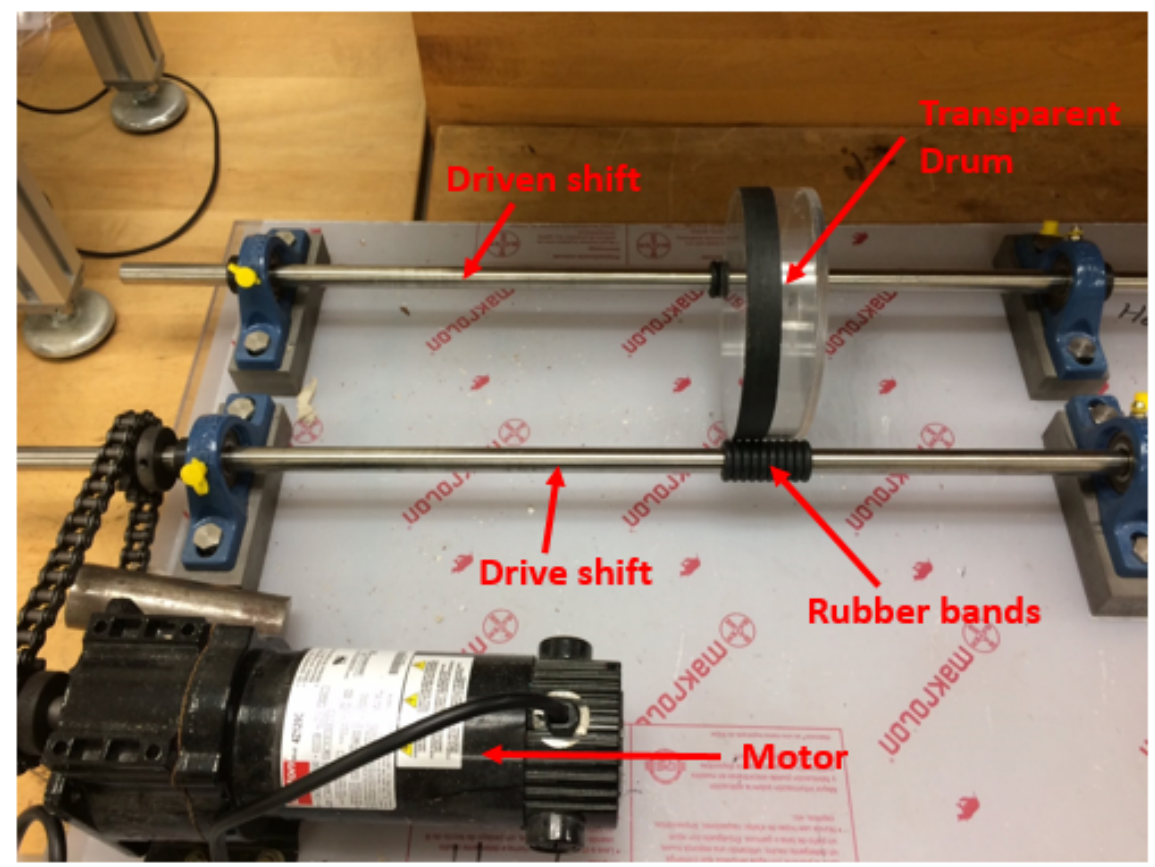

Figure 5. The rotating drum experiment setup.

Table 2. The rotating drum experiment parameters.

\begin{tabular}{ll}
\hline Parameter & Value \\
\hline Inner drum diameter $(\mathrm{mm})$ & 150 \\
Inner drum width $(\mathrm{mm})$ & 50 \\
Glass sphere diameter $(\mathrm{mm})$ & 1 \\
Filling level $(\%$ of max level depth) & 32 \\
Drum rotation speed $(\mathrm{rpm})$ & 3.26 \\
\hline
\end{tabular}

As shown in Eq. (4.5), the influence of particle diameter on the diffusion coefficient has been included explicitly and thus diffusion constants $k_{1}$ and $k_{2}$ should be independent of particle diameters. Hence, the drum was filled side by side with $1 \mathrm{~mm}$ red and blue glass spheres. To facilitate filling of the drum, the front side of the drum was made removable and a separate barrier was used to help fill each side of the drum with equal volumes of red and blue glass beads. Friction tape was used to seal the drum and to allow the container to rotate smoothly. To analyze the degree of mixing, a high-speed camera was used to film the front of the drum. Once the drum was filled, the drive shift started to rotate and the mixing process was recorded. Several snapshots of the system at different times are shown in Figure 6. Qualitatively, the mixing dynamics in the experiment followed the same trend as the simulations reported in previous work [7]. 

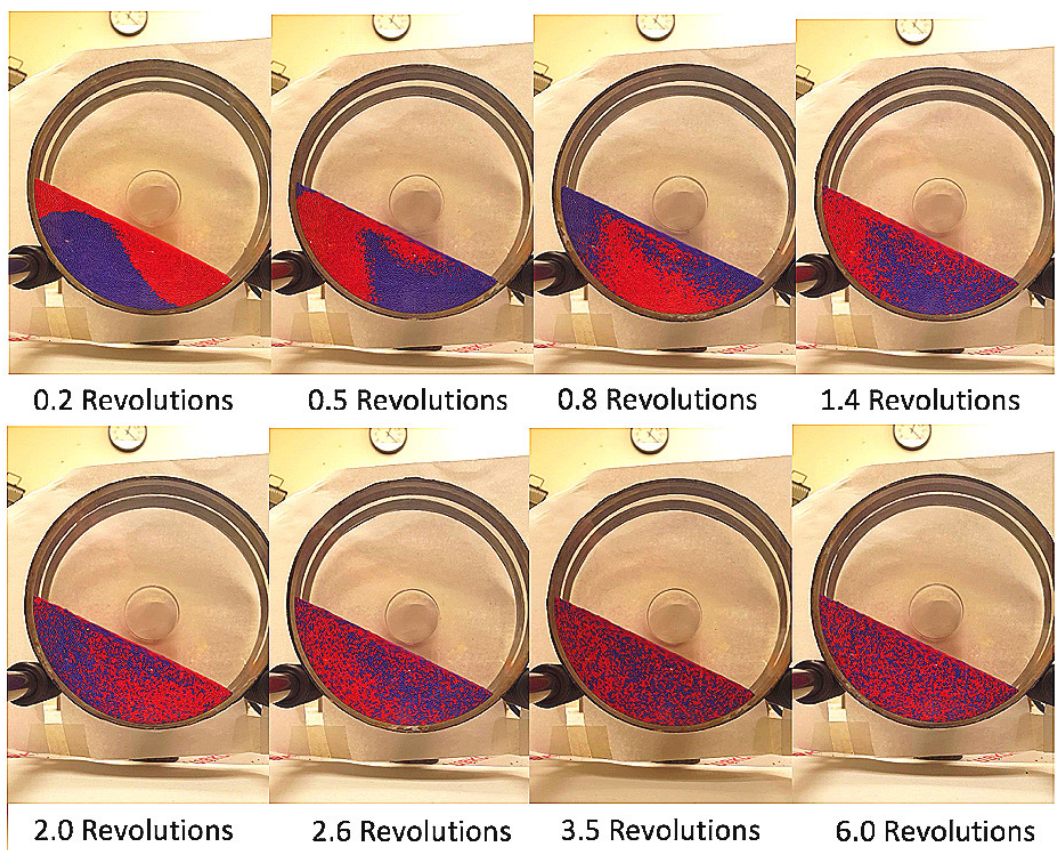

Figure 6. Snapshots showing the state of particle mixing for different numbers of drum revolutions in the rotating drum experiments.

To compute the segregation intensity as a function of the number of drum revolutions, a MATLAB code was developed to analyze the video images and derive the spatial distribution of material concentrations. Details of the image processing algorithm are included in Appendix A.

The experimental results were compared with predictions from the 2-D multi-scale model developed in the previous work [7]. To justify a comparison, the system geometry and operating conditions, such as the drum diameter, drum width, particle diameter, filling level, and rotation speed, were consistent between the experiment and the multi-scale model. The material parameters used in the FEM simulation are shown in Table 1 for glass beads.

The segregation intensity $I$ was computed to compare the experiment results with the 2-D multiscale model predictions and calibrate the spanwise diffusion constant $k_{1}$. The segregation intensity $I$ is defined as,

$$
\begin{aligned}
& I_{i}=\frac{\sigma_{\mathrm{i}}^{2}}{\sigma_{0}^{2}}, \\
& \sigma_{\mathrm{i}}^{2}=\frac{1}{M-1} \sum_{m=1}^{M}\left(c_{i}-c_{i, m}\right)^{2}, \\
& \sigma_{0}^{2}=c_{i}\left(1-c_{i}\right), \\
& c_{i}=\frac{1}{M} \sum_{m=1}^{M} c_{i, m} .
\end{aligned}
$$


In these relations, $\sigma_{i}^{2}$ is the measured variance of component $i$ 's concentration (here component $i$ is the red particles), $\sigma_{0}^{2}$ is the variance of component $i$ 's concentration for a fully segregated system, $c_{i}$ is the measured mean concentration of component $i$, and $M$ is the total number of samples used to calculate the mean and variance. The scale of scrutiny does affect the calculation, with larger scales of scrutiny having smaller segregation intensities. Hence, the same grid size used to analyze the experiment results, as described in Appendix A, was used in the 2-D multi-scale model. Computationally, the concentration values of all the nodes within one grid cell were averaged to compute the material concentration for the cell. A cell size of five particle diameters was used.

Due to the fact that a continuum is assumed in the FEM simulations, the multi-scale model would predict an asymptotic segregation intensity of nearly zero, corresponding to a perfectly mixed state. However, a perfectly mixed state is generally not achievably in practice and instead a randomly mixed state is the expected asymptotic state. For a randomly mixed system, the segregation intensity is derived as,

$I_{R} \approx \frac{\sigma_{R}^{2}}{\sigma_{0}^{2}}=\frac{1}{N}$,

where $N$ is the number of particles in the cell used to calculate the concentration. The segregation intensity for a randomly mixed system in the current work is $I=0.04$, which is shown in Figure 7 as the dashed line. To save computation time, the simulation was stopped once the segregation intensity reached the randomly mixed state.

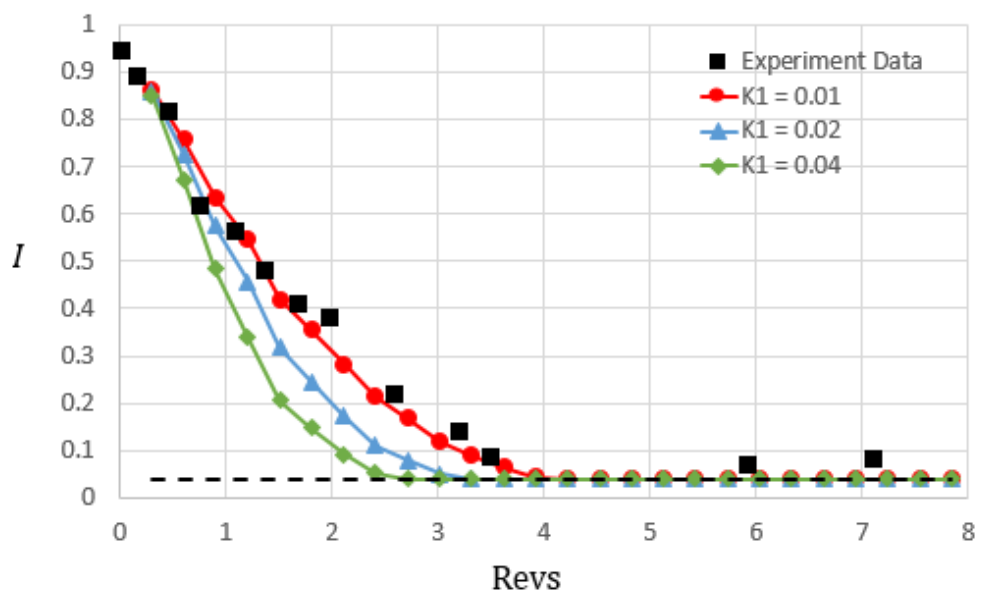

Figure 7. Segregation intensity with respect to the number of drum revolution for the experiment and the 2-D multi-scale model using different $k_{1}$ values.

Segregation intensity plotted as a function of the number of drum revolutions is plotted in Figure 7 for different assumed values of the spanwise diffusion constant $k_{1}$. Figure 8 shows the sum of the absolute differences between the segregation intensities measured from experiments and computed from the 2-D multi-scale model for different $k_{1}$ values. It can be seen that the segregation intensities computed from the 2-D multi-scale model match best with the values measured from experiments when $k_{1}=0.01$. Hence, a calibrated spanwise diffusion constant $k_{1}$ for glass beads equal to 0.01 is adopted. Note that the asymptotic value of the experimental 
results is slightly larger than the value for the randomly mixed state. This difference is because the image correction algorithm described in Appendix A introduces some error into the system and the perfectly random mixed state cannot be reached.

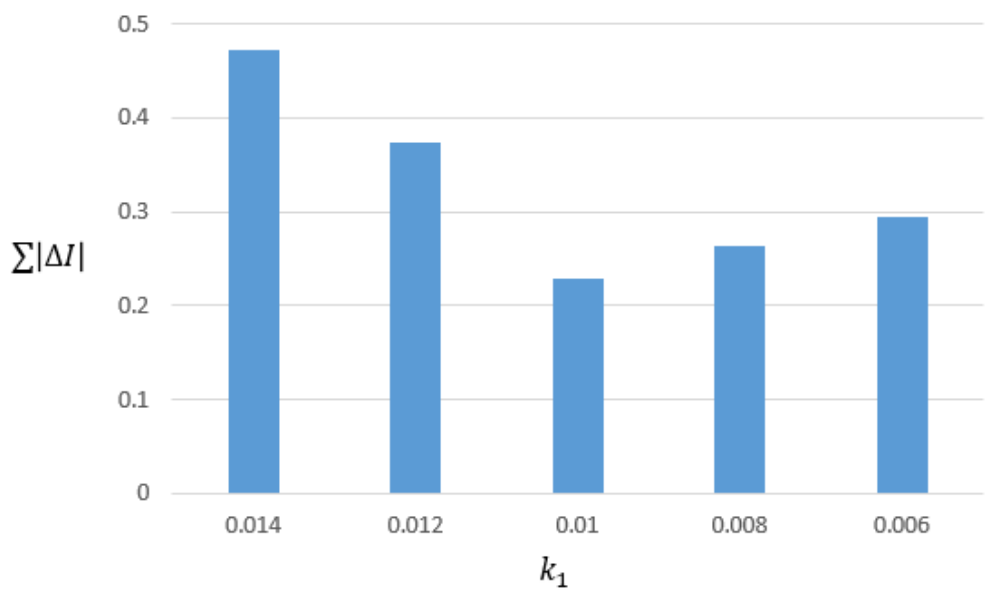

Figure 8. Sum of the absolute differences between the segregation intensities measured from experiments and computed from the 2-D multi-scale model for different $k_{1}$ values.

\section{Comparison of the experiments and multi-scale model results}

An FEM simulation was performed to predict the advective flow field information for the Sudah et al. Tote blender [26] using a rotation speed of $10 \mathrm{rpm}$ and the material properties shown in Table 1. Furthermore, since transient velocity field information was used in the current work, the velocity components in all three directions needed to be outputted at every time step, which generates considerable amounts of data. Although the velocity field is not constant within a Tote blender single revolution, the material reaches a periodic steady state condition after a few initial revolutions. Table 3 shows the averaged velocity differences between subsequent revolutions, defined as,

$$
\left|\frac{\Delta v_{i}}{v_{i}}\right|_{\text {ave }}=\frac{\sum_{n=1}^{N}\left|\frac{v_{i, n}^{j}-v_{i, n}^{j+1}}{v_{i, n}^{j}}\right|}{N},
$$

where $i$ is the direction of the velocity component, $j$ is the revolution number, and $N$ is the total number of nodes within the material domain. It is evident that the periodic steady state condition is reached after the first revolution. Therefore, to save computational effort without losing accuracy, the FEM simulation was only performed for two revolutions and the velocity fields within the second revolution were used in the multi-scale model subsequently. 
Table 3. The averaged velocity differences between subsequent revolutions.

\begin{tabular}{cccc}
\hline Revolution numbers $(\mathrm{j}, \mathrm{j}+1)$ & $(1,2)$ & $(2,3)$ & $(3,4)$ \\
\hline$\left|\Delta v_{x} / v_{x}\right|_{\text {ave }}$ & $3.95 \%$ & $0.56 \%$ & $1.85 \%$ \\
$\left|\Delta v_{y} / v_{y}\right|_{\text {ave }}$ & $2.30 \%$ & $0.38 \%$ & $3.03 \%$ \\
$\left|\Delta v_{z} / v_{z}\right|_{\text {ave }}$ & $2.91 \%$ & $1.18 \%$ & $2.53 \%$ \\
\hline
\end{tabular}

In the experiments reported by Sudah et al. [26], materials were initially loaded into the Tote blender in two different ways: top-bottom loading and left-right loading. Two filling levels were also studied $-40 \%$ fill and $60 \%$ fill. The same loading conditions and filling levels were modeled in the current work, as shown in Figure 9. Figure 10 shows the evolution of the material domain within the first revolution for left-right loading and $40 \%$ fill.

a)

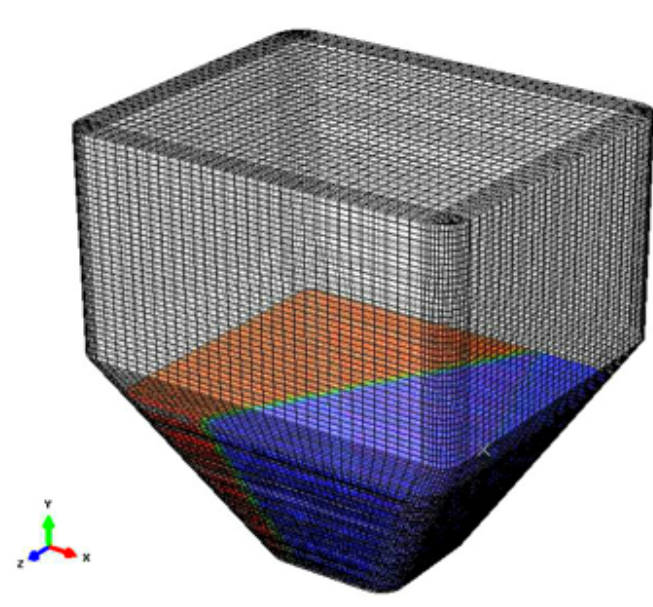

b)

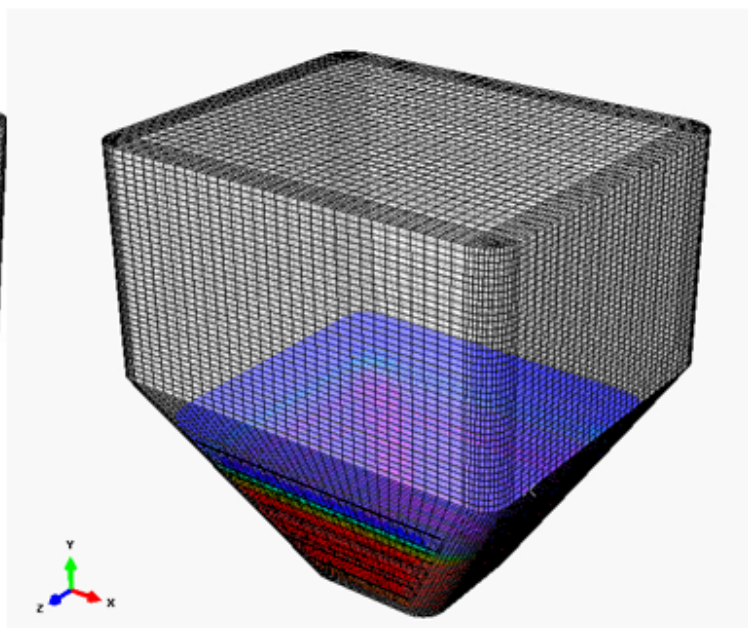

Figure 9. The left-right (a) and top-bottom (b) initial loadings for $40 \%$ fill. 
a)

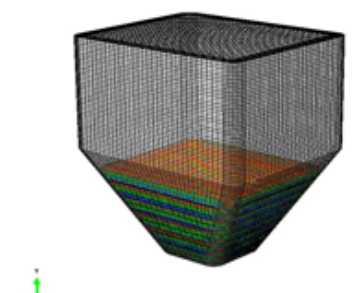

Initial

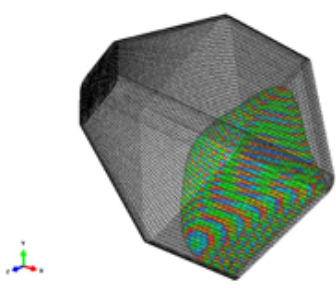

$4 \mathrm{~s}$
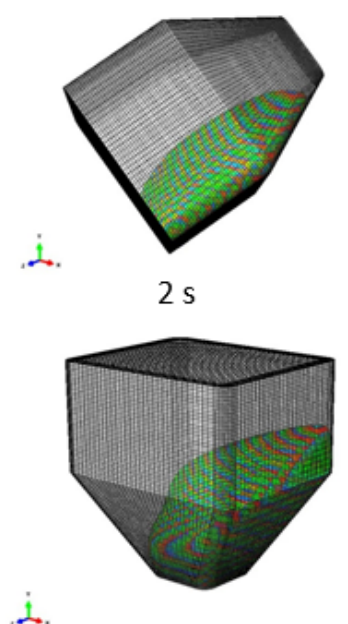

$6 s$ b)

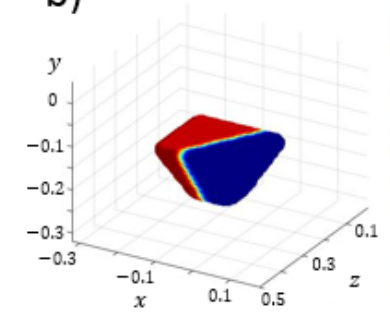

Initial

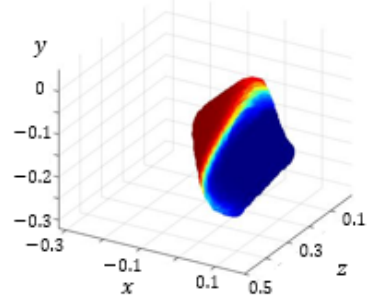

$4 \mathrm{~s}$

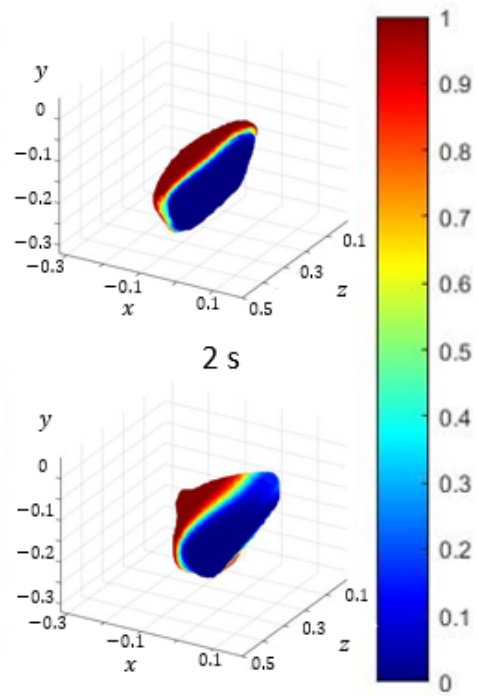

$6 \mathrm{~s}$

Figure 10. Snapshots showing the change of the material domain in the FEM simulation (a) and multi-scale model (b) for left-right loading and $40 \%$ fill. The vertical color scale in (b) is the red particle concentration.

The state of mixing predicted by the multi-scale model after different numbers of revolutions is shown in Figure 11. As expected, as time increases the degree of mixing increases, with both advection and diffusion contributing to the mixing process. Moreover, the top-bottom loaded materials mix much faster than the left-right loaded materials since the advective mixing is much stronger in the top-bottom loading case.

a)

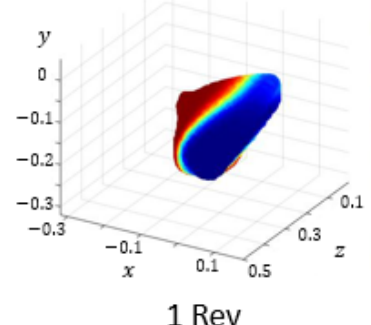

1 Rev

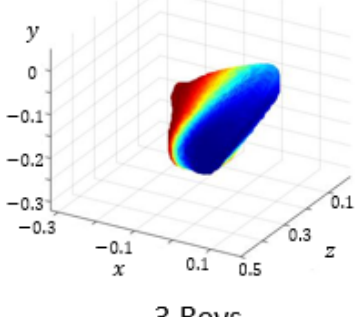

3 Revs

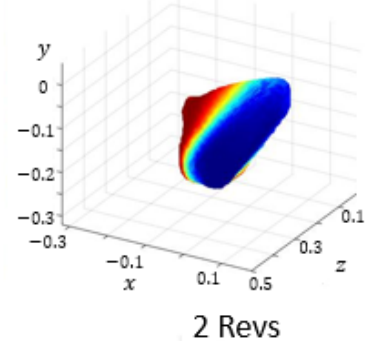

2 Revs

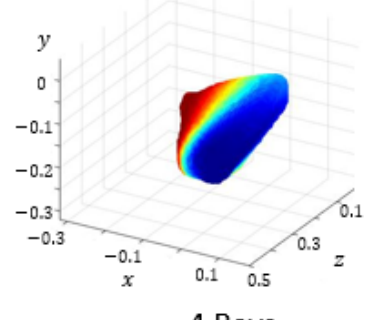

4 Revs b)
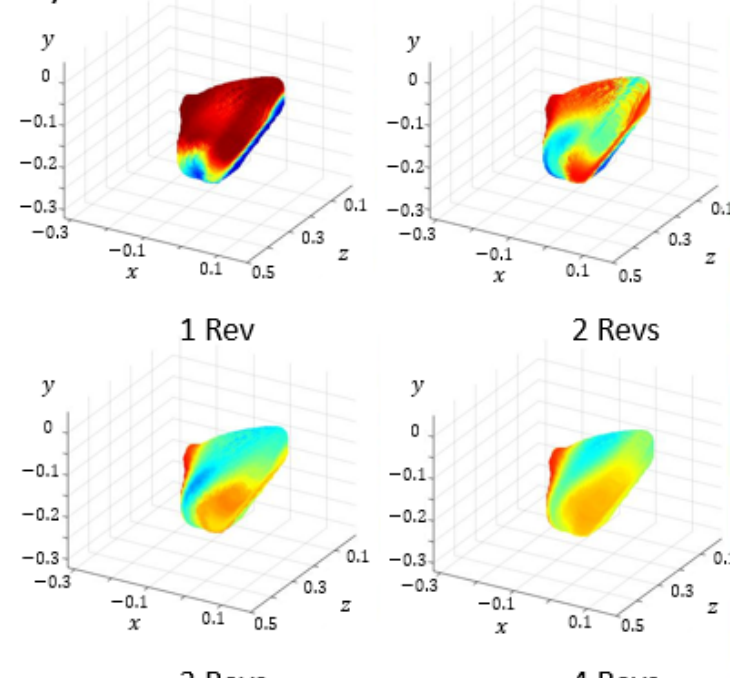

3 Revs

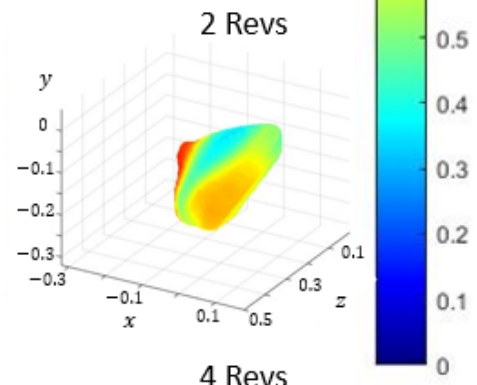

4 Revs

Figure 11. Snapshots showing the state of mixing at the end of each revolution for (a) left-right loading and (b) top-bottom loading and $40 \%$ fill. The vertical color scale is the red particle concentration for (a) and (b). 
In the experiments by Sudah et al. [26], mixing curves were constructed by plotting the relative standard deviation $(R S D)$ as a function of mixing time. The relative standard deviation is calculated as,

$$
\begin{aligned}
& R S D=\frac{\sigma}{c_{i}}, \\
& \sigma^{2}=\frac{1}{M-1} \sum_{m=1}^{M}\left(c_{i}-c_{i, m}\right)^{2} .
\end{aligned}
$$

In the above equations, $\sigma^{2}$ is the measured variance of component $i$ 's concentration, $c_{i}$ is the mean concentration of component $i$, and $M$ is the total number of samples used to calculate the mean and variance. The relative standard deviation varies from zero, corresponding to perfect mixing, to one, which is a fully segregated state. The $R S D$ value was also computed using the multi-scale model to compare with the published experimental results. Note that in the multiscale model, every node at which a concentration is calculated is used in the evaluation of the segregation intensity, while in the experiments nine core samples were used [26]. Although the scale of scrutiny plays a role in the calculated results, as shown in previous work [7], the predicted values using different grid sizes are close to each other.

Figure 12 plots the $R S D$ values with respect to the number of revolutions for top-bottom and leftright initial loading patterns for the multiscale-model and experiments. Results for $40 \%$ and $60 \%$ fill levels were compared. In the current work, one million cells were used to maintain accuracy and computational efficiency (i.e., 100 cells in each direction). A mesh dependency study was performed to ensure solution convergence and the error was found to be within $5 \%$ between one million cells (i.e., 100 cells in each direction) and 3.375 million cells (i.e., 150 cells in each direction). As shown in Figure 12, there is good quantitative agreement between the multi-scale model predictions and experimental measurements, although it does appear that there is some offset for the case with $40 \%$ fill and top-bottom loading. There is no error information given for the experimental results so it is difficult to determine how significant this difference is. The total wall-clock time required to run a single case on 10 cores, including the FEM simulation and MATLAB processing, was approximately 2.5 days. 

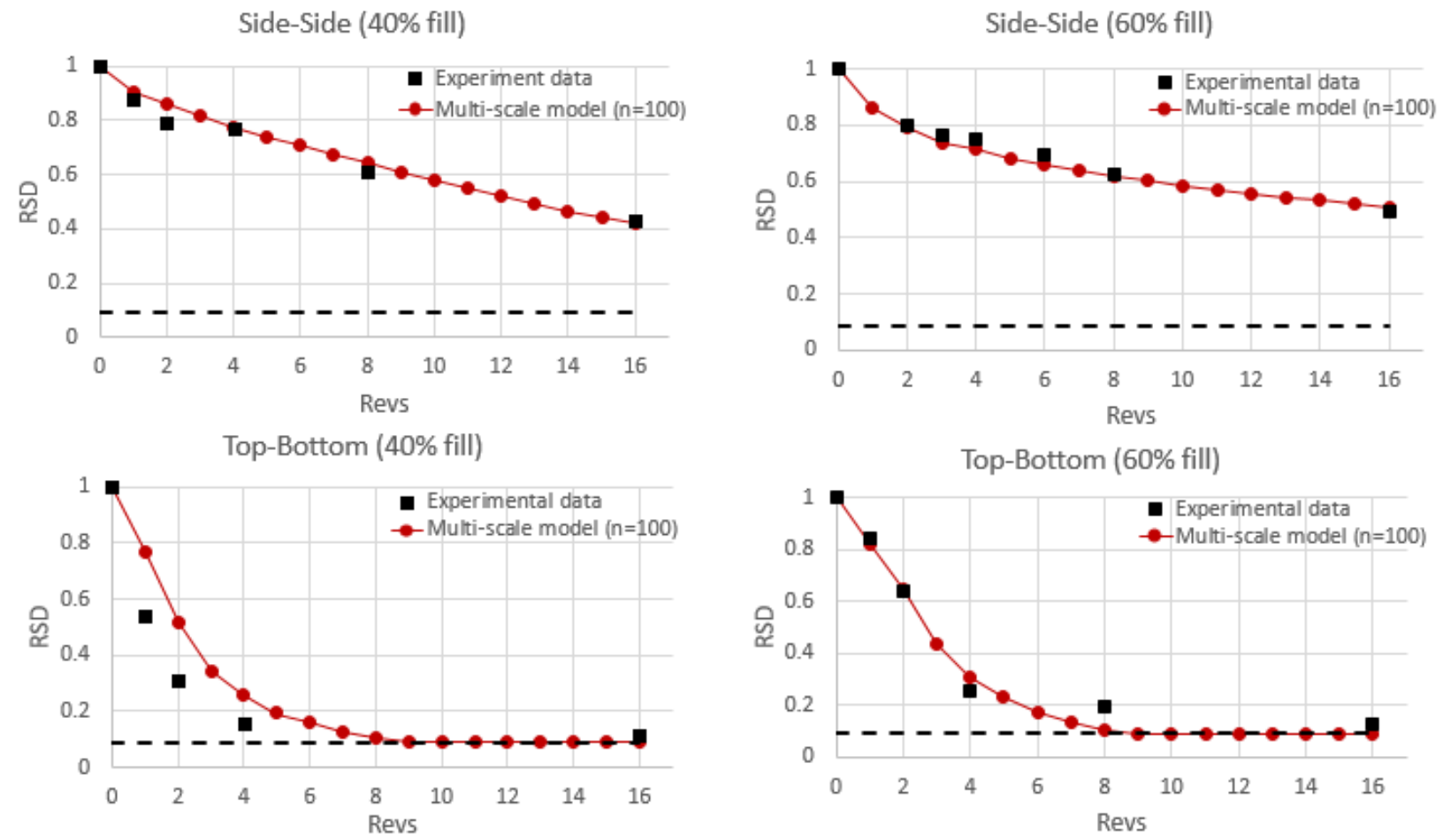

Figure 12. Mixing state comparison between published experimental results and the multi-scale model predictions. The random mixing state is represented by horizontal dashed lines and the spanwise diffusion constant is $k_{1}=0.01$.

\section{Conclusions}

In this work, a three-dimensional transient multi-scale modeling approach is described for predicting blending in particulate systems. This model is extended from a previously published two-dimensional steady model [7]. This three-dimensional model combines, within the advection-diffusion equation, finite element method generated transient macroscopic velocity fields with experimentally-obtained particle diffusion correlations at a local scale. The model is applied here to a three-dimensional Tote blender. Predictions of the mixing rate from the multiscale model compare well quantitatively to published experimental data [26].

A significant advantage of the multi-scale blending model over a DEM-only model is that the multi-scale model is more computationally efficient for industrially-relevant system sizes. This is because the number of DEM particles increases with the cube of the ratio of the system size to particle size, while the FEM nodes do not necessarily need to increase since the mesh size increases linearly with the system size. Furthermore, if particle size is reduced in the DEM simulations, then the integration time step also decreases, further increasing the time required to complete DEM simulations. In addition, all of the parameters used in the multi-scale model can be measured from independent, standard tests or calibrated from simple two-dimensional experiments (such as the material internal friction angle, the wall friction angle, and the diffusion constant). No back-fitting of the Tote blender results was used in the current work. 
Future work should focus on expanding the multi-scale model to consider segregation of different materials, which is important in industrial practice. In addition, a simple, standard method should be developed to calibrate the diffusion constants. The current work used a twodimensional blender experiment, but other systems, such as a 2-D heap flow, may be considered.

\section{Appendix A Image Processing algorithm}

A flowchart of the image processing algorithm used in the current work is shown in Figure A1. For each image, the material domains (red and blue glass beads) were extracted using the freeware program ImageJ [35], which was used to identify only the glass beads among a white background. This RGB image was further analyzed to extract only the red component of each image's pixels, with a value ranging between 0 (no red) and 255 (all red). A threshold value of 80 was selected to differentiate between the red and blue pixels. A value larger than 80 indicated that a pixel corresponded to a red particle while a value smaller than 80 indicated that it was a blue particle. This threshold value was chosen to ensure that at any point in time the fraction of red pixels in the entire system was $50 \pm 5 \%$ since the system consisted of $50 \%$ red beads.

1

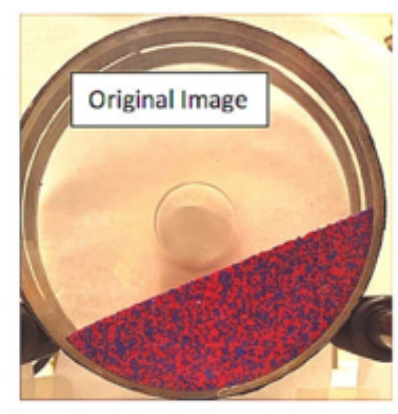

4

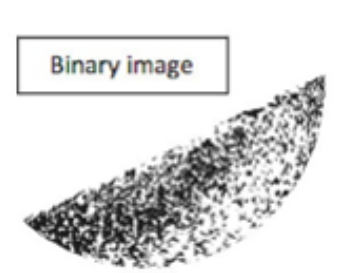

2

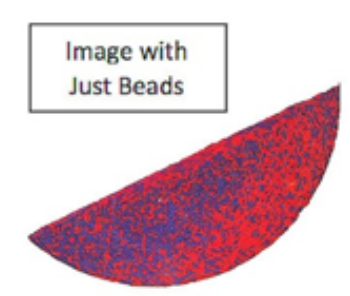

5

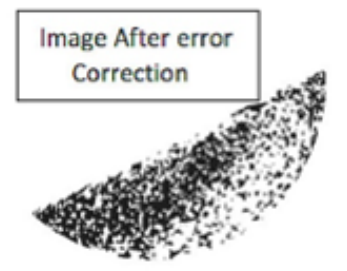

3

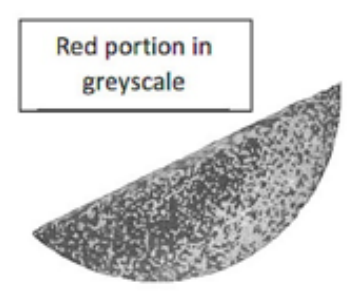

Figure A1. The image analysis algorithm for a single image. The steps proceed from 1 to 5 .

After distinguishing red and blue pixels, a black and white binary image was generated, with black corresponding to the blue pixels and white corresponding to the red ones. Note that since spherical glass beads were used in the experiment, reflections and shadows were introduced due to the light source. An image correction algorithm was developed in the MATLAB program in an attempt to account for these effects.

The corrected binary image was used to compute the segregation intensity. A non-overlapping grid of square cells was used in the current analysis. The cell size varied from 3 to 10 particle diameters on a side. To avoid using samples containing data outside the cylinder boundary, boundary flags were used to make sure each sample contained at least $95 \%$ material. All of the qualified samples were then used to calculate the segregation intensity for the current time step. 


\section{References}

[1] L.T. Fan, S.J. Chen, C.A. Watson, Solids Mixing, Ind. Eng. Chem. 62 (1970) 53-69. doi:10.1021/ie50727a009.

[2] M. Poux, P. Fayolle, J. Bertrand, D. Bridoux, J. Bousquet, Powder mixing: Some practical rules applied to agitated systems, Powder Technol. 68 (1991) 213-234. doi:10.1016/00325910(91)80047-M.

[3] M. Moakher, T. Shinbrot, F.J. Muzzio, Experimentally validated computations of flow, mixing and segregation of non-cohesive grains in 3D tumbling blenders, Powder Technol. 109 (2000) 58-71. doi:10.1016/S0032-5910(99)00227-2.

[4] Z. Asaf, D. Rubinstein, I. Shmulevich, Determination of discrete element model parameters required for soil tillage, Soil Tillage Res. 92 (2007) 227-242. doi:10.1016/j.still.2006.03.006.

[5] G.K.P. Barrios, R.M. de Carvalho, A. Kwade, L.M. Tavares, Contact parameter estimation for DEM simulation of iron ore pellet handling, Powder Technol. 248 (2013) 84-93. doi:10.1016/j.powtec.2013.01.063.

[6] C.J. Coetzee, D.N.J. Els, G.F. Dymond, Discrete element parameter calibration and the modelling of dragline bucket filling, J. Terramechanics. 47 (2010) 33-44. doi:10.1016/j.jterra.2009.03.003.

[7] Y. Liu, M. Gonzalez, C.R. Wassgren, Modeling granular material blending in a rotating drum using a finite element method and advection- diffusion equation multi-scale model Yu Liu, Https://Arxiv.Org/Pdf/1704.01219. (2017) 1-24.

[8] A. Sarkar, C.R. Wassgren, Effect of particle size on flow and mixing in a bladed granular mixer, AIChE J. 61 (2015) 46-57. doi:10.1002/aic.14629.

[9] K. Yamane, Discrete-element method application to mixing and segregation model in industrial blending system, J. Mater. Res. 19 (2004) 623-627.

doi:10.1557/jmr.2004.19.2.623.

[10] P.Y. Liu, R.Y. Yang, A.B. Yu, DEM study of the transverse mixing of wet particles in rotating drums, Chem. Eng. Sci. 86 (2013) 99-107. doi:10.1016/j.ces.2012.06.015.

[11] R. Chandratilleke, A. Yu, J. Bridgwater, K. Shinohara, Flow and mixing of cohesive particles in a vertical bladed mixer, Ind. Eng. Chem. Res. 53 (2014) 4119-4130. doi:10.1021/ie403877v.

[12] J. Bridgwater, D.F. Bagster, S.F. Chen, J.H. Hallam, Geometric and dynamic similarity in particle mixing, Powder Technol. 2 (1969) 198-206. doi:10.1016/0032-5910(69)80013-6.

[13] J. Bridgwater, W.S. Foo, D.J. Stephens, Particle mixing and segregation in failure zonestheory and experiment, Powder Technol. 41 (1985) 147-158. doi:10.1016/00325910(85)87033-9.

[14] S.B. Savage, C.K.K. Lun, Particle size segregation in inclined chute flow of dry cohesionless granular solids, J. Fluid Mech. 189 (1988) 311-335. doi:10.1017/S002211208800103X.

[15] J.M.N.T. Gray, V.A. Chugunov, Particle-size segregation and diffusive remixing in shallow granular avalanches, 2006. doi:10.1017/S0022112006002977.

[16] Y. Fan, C.P. Schlick, P.B. Umbanhowar, J.M. Ottino, R.M. Lueptow, Modelling size segregation of granular materials: The roles of segregation, advection and diffusion, $\mathrm{J}$. Fluid Mech. 741 (2014) 252-279. doi:10.1017/jfm.2013.680. 
[17] C.P. Schlick, Y. Fan, P.B. Umbanhowar, J.M. Ottino, R.M. Lueptow, Granular segregation in circular tumblers: Theoretical model and scaling laws, J. Fluid Mech. 765 (2015) 632-652. doi:10.1017/jfm.2015.4.

[18] D. Bertuola, S. Volpato, P. Canu, A.C. Santomaso, Prediction of segregation in funnel and mass flow discharge, Chem. Eng. Sci. 150 (2016) 16-25. doi:10.1016/j.ces.2016.04.054.

[19] S.K. Hajra, D. Shi, J.J. McCarthy, Granular mixing and segregation in zigzag chute flow, Phys. Rev. E - Stat. Nonlinear, Soft Matter Phys. 86 (2012) 1-7. doi:10.1103/PhysRevE.86.061318.

[20] L. Bai, Q.J. Zheng, A.B. Yu, FEM simulation of particle flow and convective mixing in a cylindrical bladed mixer, Powder Technol. 313 (2017) 175-183.

doi:10.1016/j.powtec.2017.03.018.

[21] Q.J. Zheng, A.B. Yu, Modelling the granular flow in a rotating drum by the Eulerian finite element method, Powder Technol. 286 (2015) 361-370.

doi:10.1016/j.powtec.2015.08.025.

[22] Q.J. Zheng, A.B. Yu, Finite element investigation of the flow and stress patterns in conical hopper during discharge, Chem. Eng. Sci. 129 (2015) 49-57. doi:10.1016/j.ces.2015.02.022.

[23] S. Swaminathan, J. Hilden, B. Ramey, C. Wassgren, Modeling the Formation of Debossed Features on a Pharmaceutical Tablet, J. Pharm. Innov. 11 (2016) 214-230. doi:10.1007/s12247-016-9257-6.

[24] S. Swaminathan, B. Ramey, J. Hilden, C. Wassgren, Characterizing the powder punchface adhesive interaction during the unloading phase of powder compaction, Powder Technol. 315 (2017) 410-421. doi:10.1016/j.powtec.2017.04.003.

[25] Y. Liu, C. Wassgren, Modifications to Johanson's roll compaction model for improved relative density predictions, Powder Technol. 297 (2016) 294-302. doi:10.1016/j.powtec.2016.04.017.

[26] O.S. Sudah, P.E. Arratia, A. Alexander, F.J. Muzzio, Simulation and experiments of mixing and segregation in a tote blender, AIChE J. 51 (2005) 836-844. doi:10.1002/aic.10448.

[27] Dassault Systèmes, Abaqus analysis user's manual, Rhode Island, 2007.

[28] B. Utter, R.P. Behringer, Self-diffusion in dense granular shear flows, Phys. Rev. E - Stat. Nonlinear, Soft Matter Phys. 69 (2004) 1-12. doi:10.1103/PhysRevE.69.031308.

[29] T.J. Chung, Computational Fluid Dynamics, Cambridge University Press, Cambridge, 2015.

[30] Y. Liu, X. Guo, H. Lu, X. Gong, An investigation of the effect of particle size on the flow behavior of pulverized coal, Procedia Eng. 102 (2015) 698-713. doi:10.1016/j.proeng.2015.01.170.

[31] E.M. Kara, Contribution of particles size ranges to sand friction, Eng. Technol. Appl. Sci. Res. 3 (2013) 497-501.

[32] F. Podczeck, Y. Miah, The influence of particle size and shape on the angle of internal friction and the flow factor of unlubricated and lubricated powders, Int. J. Pharm. 144 (1996) 187-194. doi:10.1016/S0378-5173(96)04755-2.

[33] A. Castellanos, C. Soria-Hoyo, J.M. Valverde, M.A.S. Quintanill, Cohesion and internal friction of fine glass beads as affected by small intensity vertical vibration, AIP Conf. Proc. 1145 (2009) 707-710. doi:10.1063/1.3180025.

[34] N. Mitarai, F. Nori, Wet granular materials, Adv. Phys. 55 (2006) 1-45. 
doi:10.1080/00018730600626065.

[35] C.A. Schneider, W.S. Rasband, K.W. Eliceiri, NIH Image to ImageJ : 25 years of image analysis HISTORICAL commentary NIH Image to ImageJ : 25 years of image analysis, Nat. Methods. 9 (2012) 671-675. doi:10.1038/nmeth.2089. 